\title{
Hospital Parroquial de San Bernardo, Chile: 115 años sirviendo a la comunidad
}

\author{
RICARDO ESPINOZA G.
}

\section{Parochial hospital of San Bernardo, Chile. Serving the community for over a century}

The Parochial Hospital of San Bernardo is an intermediate complexity hospital that functions in its original 115-year-old building. It is one of the oldest hospitals in the country. Driven by the local Catholic Church and despite multiple difficulties, the hospital has uninterruptedly served a progressively growing community, with medical care and spiritual support. In the last two decades, it also has incorporated teaching activities with Universidad de los Andes Medical School.

(Rev Med Chile 2013; 141: 119-122).

Key words: Community health services; Education, Medical; Hospitals, Community.

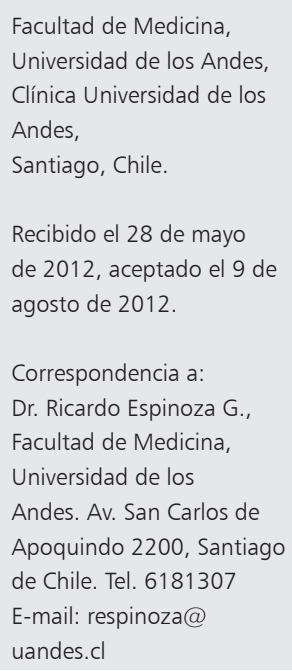

L a época colonial en Chile concluía con diez establecimientos hospitalarios a lo largo del territorio, entre Arica y Valdivia. La administración y la labor asistencial propiamente tal estaban a cargo de una orden religiosa y por ello, anexos al respectivo hospital, habitualmente existían un convento y una iglesia. Posteriormente, se traspasó el control de los hospitales a los municipios y, con el aporte constante de donaciones de privados sumado a los esfuerzos gubernamentales, se fue desarrollando gradualmente la asistencia hospitalaria durante el siglo XIX. Hay que añadir la llegada de nuevos médicos que, de ser no más de 20 a comienzos del siglo llegaron a unos 200 al finalizar la centuria. Hacia 1885, cuando la población del país bordeaba los 2,5 millones de habitantes, existían 60 hospitales en Chile. Santiago, con unos 200.000 habitantes, contaba con seis hospitales, destacando el Hospital San Juan de Dios y el Hospital San Francisco de Borja; el número total de camas se empinaba a unas mil, empero se requería urgentemente mejorar las condiciones sanitarias; las elevadas tasas de natalidad pero también de mortalidad, requerían renovados esfuerzos para crear nuevos recintos hospitalarios ${ }^{1}$. Así, en los últimos decenios del siglo XIX grandes movilizaciones permitieron la construcción del
Hospital del Salvador y el Hospital San Vicente de Paul, constituyéndose este último en el primer hospital clínico universitario.

\section{Condiciones locales de la Villa de San Bernardo}

No obstante, en la Villa de San Bernardo, que distaba cuatro leguas de Santiago, hasta 1887 existía sólo un dispensario, con un "flebótomo" como todo recurso profesional, que en el diario local ofrecía así sus servicios: "estracciones, limpiadura de dientes, ventosas mojadas, sangrías y demás convenientes a la profesión”. San Bernardo se había constituido en lugar de descanso y veraneo de pudientes familias santiaguinas y su población estable alcanzaba a unos 10.000 habitantes. El traslado a Santiago en un tranvía "de sangre" (con tracción animal, por caballo) podía tomar horas: urgía la creación de un hospital local ${ }^{2}$.

La población se movilizó y recurrió a la autoridad eclesiástica en busca de ayuda. Se recolectaron fondos y con la autorización del Arzobispado de Santiago, el 3 de marzo de 1887 el Presbítero y Vicario de San Bernardo, Don José Manuel Duozorroza, firmó la escritura de adquisición de 
los terrenos del futuro hospital, pagándose la suma de \$4.200. La primera piedra fue colocada un año más tarde.

Se conformó una comisión encargada de desarrollar el proyecto, siendo su principal impulsor Monseñor José Joaquín Larraín Gandarillas, quien pasaba algunas temporadas en la Villa, aquejado de tuberculosis. Con todo, los fondos que se lograba reunir eran insuficientes y se avanzaba con lentitud, sin que se materializara el anhelado hospital. Hay que sumar la compleja situación nacional derivada de la Revolución de 1891, cuando comenzaron a llegar a San Bernardo heridos de las batallas; los primeros fueron atendidos en la misma casa del Obispo y luego en un precario recinto en la calle Colón esquina con Urmeneta, donde se habían ubicado: "quince camas, arregladas $y$ distribuidas en tres espaciosas y ventiladas salas, una de cirugía y dos de enfermedades internas", donde los enfermos y heridos eran atendidos por religiosas, el doctor Gómez Martínez y el practicante señor Olivares ${ }^{2}$.

En abril de 1895 el Arzobispo de Santiago decretó como administrador del Hospital al Obispo Larraín Gandarillas y a don Macario Ossa Cerda, Gobernador, como viceadministrador. Donaciones de la familia Ducaud permitieron contar con nuevos terrenos para la construcción del establecimiento definitivo y así, dos años después, en 1897 se pudo habilitar gran parte del edificio que permanece hasta la actualidad.

\section{El naciente Hospital Parroquial de San Bernardo}

La construcción del hospital fue obra, en parte, del arquitecto Juan Izquierdo, "conjunto de dos pisos, de albañilería y adobe, en torno a patios interiores, porticados y con galerías"”. La administración fue encomendada a la Congregación de las Hijas de San José, quienes en número de cinco, asumieron la responsabilidad en octubre de 1897. La renta de cada religiosa era de 15 pesos al mes. El Hospital estaba preparado para recibir hasta 35 pacientes. Cuatro años más tarde, en 1901, se designó como médico oficial del estable- cimiento al doctor Federico Puga Borne, quien debía atender, además, el dispensario anexo. Luego se sumó el doctor Santiago Mac-Lean. Con don Macario Ossa a la cabeza, nuevos aportes del gobierno permitieron la construcción de más salas, para albergar ochenta enfermos. De este modo, en el primer cuerpo del edificio, con cinco patios, se ubicaba la Capilla y dependencias del capellán, la sala de hospitalización de mujeres y las habitaciones de las religiosas y oficinas ${ }^{2}$. En el segundo cuerpo se situaban las habitaciones para los hombres, la botica y la sala de operaciones (Figura 1).

Así, poco a poco, el Hospital aumentaba sus recursos y capacidad para responder a las necesidades habituales de la población en situaciones cotidianas y ante condiciones de excepción, como lo fue la epidemia de viruela en 1904 y el terremoto de 1906. La Capilla del Hospital, dañada por el sismo, fue reconstruida por el arquitecto Eugenio Joannon Crozier, en estilo neogótico ${ }^{2}$; los trabajos duraron una década y se hicieron con recursos provenientes de economías hechas en el hospital y del trabajo de las religiosas (Figura 2).

Recién en 1932 se pudo contar con una maternidad, siendo el doctor Luis Sepúlveda Salvatierra el Director del Hospital y las religiosas de la Congregación Hijas de Santa Ana, Madre de María Inmaculada, quienes colaboraban en la asistencia. Se comenzó con 10 camas para la atención de las parturientas ${ }^{2}$.

La constante falta de recursos fue siempre la tónica de esta institución de la Iglesia; recibía exiguos fondos del gobierno y un tanto por el arriendo de una propiedad en San Bernardo, pero las sumas más importantes provenían de donaciones de los vecinos más pudientes.

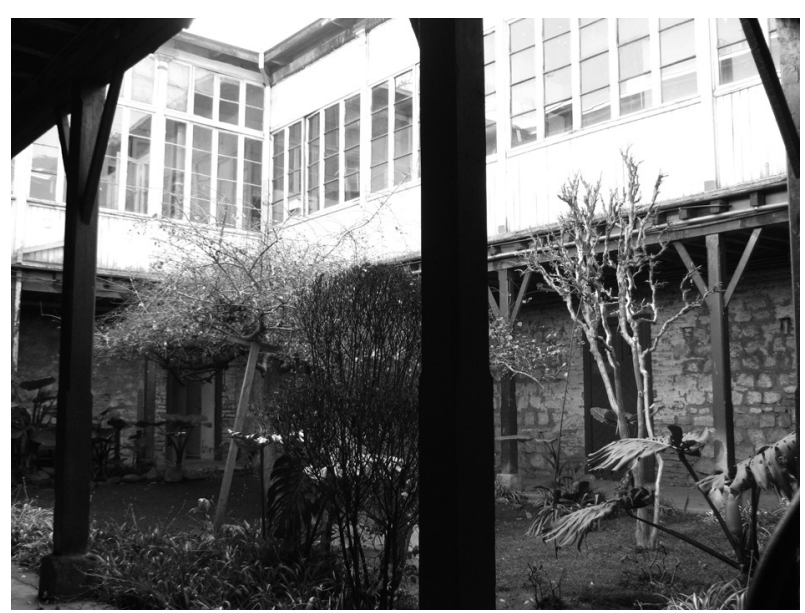

Figura 1. Uno de los patios interiores del Hospital Parroquial de San Bernardo. 


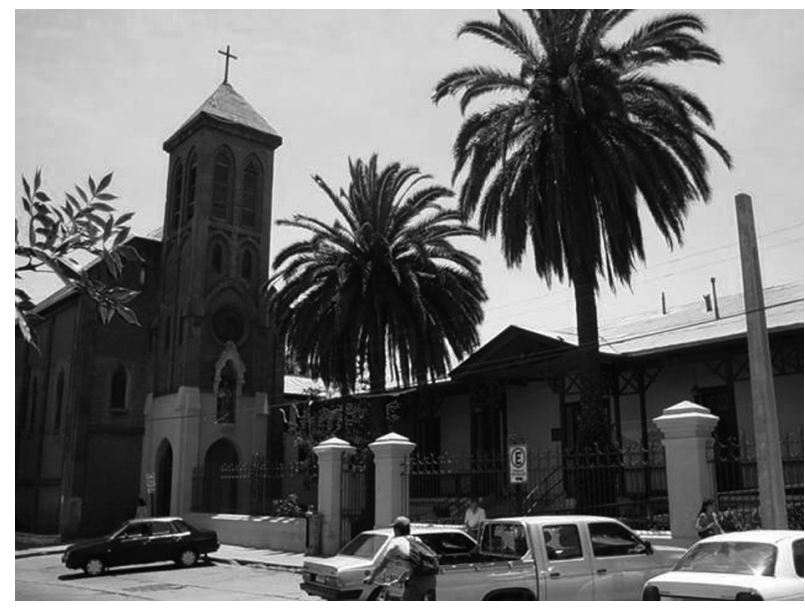

Figura 2. Fachada principal del hospital y su Capilla.

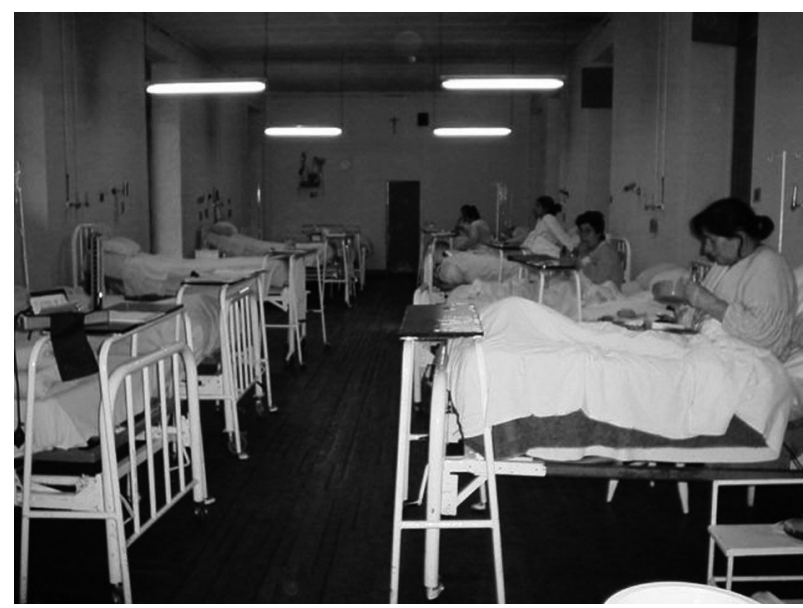

Figura 3. Sala de Cirugía de Mujeres, hacia comienzos del año 2002.

Con todo, las religiosas realizaban una colecta anual y un bazar, donde vendían tejidos y bordados hechos por ellas mismas, lo que permitía alimentar a los enfermos. En este trabajo destacó la actuación de Sor Cunegunda Margutti, italiana, quien fue distinguida por el gobierno de la República con la Orden de Bernardo O’Higgins, por sus servicios a la comunidad. A partir de 1940 se logró una mayor estabilidad, cuando se estableció un convenio con la Empresa de Ferrocarriles del Estado, la que entregaba ayuda en ropa, calefacción, electricidad y mantención a cambio de los servicios asistenciales a los funcionarios de su Maestranza. Cuando no había calefacción se debía abrigar muy bien a los enfermos y se recurría a estufas de aserrín. Con todo, las dependencias del hospital eran muy frías. Cuenta uno de los cirujanos de entonces que "en invierno salían grandes cantidades de vapor cuando se abría el abdomen de los operados" adormecidos bajo los vapores de éter administrados por un inhalador de Ombredanne, siendo las mismas religiosas quienes actuaban como anestesistas y ayudantes ${ }^{2}$. Entre 1945 y 1955 ya se habían incorporado en forma estable al trabajo del hospital los doctores César Avaria, ginecólogo y los cirujanos Luis Sepúlveda Dagnino, Guillermo Guzmán Valenzuela y Patricio Silva Garín.

Además, si bien el Hospital era de adultos, hasta la década de 1950 en él vivían unos 80 niños, "entre enfermos del pulmón, huérfanos y abandonados".

\section{Historia reciente del Hospital}

En las décadas de 1960 y 1970, la carestía volvía a golpear al hospital. Los ingresos provenían de una pequeña cantidad por cada enfermo atendido que perteneciera a la Caja de Seguro Obrero, ingresos del todo insuficientes. Más tarde se estableció un convenio con el Servicio de Salud Metropolitano Sur, que hasta la actualidad ha delegado en el hospital la atención de pacientes de una determinada área geográfica, derivados desde Consultorios de Salud Primaria.

Hacia 1975 la situación se había hecho económicamente insostenible, por lo que se constituyó la Fundación de Beneficencia Hospital Parroquial de San Bernardo para agilizar y hacer más eficiente su administración. Así, el hospital se podía adaptar mejor a las condiciones del momento. Luego su dependencia fue traspasada de la Parroquia de San Bernardo, perteneciente al Arzobispado de Santiago, al recién creado Obispado de San Bernardo. De este modo, hoy la Fundación la dirige el señor Obispo de la Diócesis, quien administra el hospital con un Directorio. En años recientes son variadas las instituciones y fundaciones que han hecho donaciones al hospital, permitiendo modernizar el Laboratorio, crear una moderna Unidad de Neonatología, ampliar la Unidad de Cuidados Intensivos, remodelar los Servicios de Ginecología y Obstetricia y el de Urgencia. 
En la actualidad el hospital cuenta con 155 camas y todos los servicios clínicos de un hospital general. El año 2011 se registraron 8.229 egresos y 1.764 partos, actividad que se efectúa, mayoritariamente, en personas indigentes. (Figura 3). Desde el año 2005 se han incorporado al hospital los Padres Camilianos, revitalizando con sus actividades la asistencia espiritual a los pacientes internados ${ }^{4}$.

\section{Incorporación de la docencia}

Quien fuera el fundador del hospital, Monseñor Larraín Gandarillas, siempre anheló que el Hospital Parroquial de San Bernardo cumpliera una doble misión: por un lado, que diera atención médica con caridad cristiana a los con menos recursos y, por otro, que fuese un centro formador de futuros médicos. Esto último llegó en 1985, cuando fue firmado un convenio con la Universidad Católica de Chile, para ser uno de sus campos clínicos. Se restructuró la Dirección del hospital y se inició la docencia con alumnos de las carreras de Medicina y de Enfermería, experiencia que duró tres años ${ }^{2}$. Fue un período de adelantos y proyecciones.

Luego, en 1990, el entonces Obispo de San Bernardo, Monseñor Orozimbo Fuenzalida y el Rector de la Universidad de los Andes, don Raúl Bertelsen R. firmaron un Convenio DocenteAsistencial de carácter exclusivo que se mantiene hasta la actualidad. Gracias a este acuerdo, ya son más de veinte las promociones de médicos y enfermeras de esta Universidad que han completado su formación en los recintos del hospital, brindando cuidado y aportando sus conocimientos. Actualmente, funciona anexo al hospital un Centro de Salud Ambulatoria de la Universidad de los Andes, donde se realiza docencia teórica y práctica para las carreras de Medicina, Enfermería, Odontología, Psicología y Kinesiología.

En palabras de autoridades locales, el Hospital Parroquial se ha convertido en un patrimonio de la Comuna de San Bernardo y seguirá sirviendo a quienes acudan a él, con la misma vocación que tuviera en sus inicios, hace ya 115 años. Su aniversario de fundación se celebra cada 15 de noviembre.

\section{Referencias}

1. Cruz-Coke R. Historia de la Medicina Chilena. Editorial Andrés Bello. Santiago, Chile, 1995.

2. Pearl E. 100 años. Hospital Parroquial de San Bernardo. Escuela de Periodismo, Universidad de los Andes. Santiago, Chile, 1997.

3. Guía Patrimonial de San Bernardo. Master en Historia y gestión del patrimonio cultural, Universidad de los Andes. Santiago, Chile, 2008.

4. Revista de la Iglesia de San Bernardo. Obispado de San Bernardo, febrero 2007. 